\title{
Proteomic analysis of immunocamouflaged surfaces
}

The transfusion of red blood cells (RBC) is a critical component in the treatment of a number of acute and chronic medical problems. Indeed, approximately 75 million units of whole blood ( 34 million liters) are annually collected worldwide for processing and eventual transfusion. Despite this massive collection effort, the need for blood constantly exceeds availability due to a combination of collection, manufacturing, storage and biological (i.e., immunological) issues. The immunological issues can often be the most clinically vexing as the RBC has $~ 300$ blood group antigens that could potentially result in alloimmunization and, in rare cases, prevent life saving transfusions. Currently, there are limited options to either prevent blood group alloimmunization or to cost-effectively treat patients with severe alloimmunization.

To this end, the bioengineering of RBCs by the grafting of biocompatible polymers, such as methoxypoly(ethylene glycol) (mPEG), to donor cells is a promising non-pharmacological immunomodulation technology for preventing RBC transfusion reactions. However, due to the labile nature of RBCs, surface-plasma interactions are poorly understood. To overcome the limitations imposed by viable cells, Dr. Mark Scott and his colleagues at the Canadian Blood Services and the University of British Columbia examined plasma adsorption to bare and polymer modified latex beads using fluorescently labeled plasma and proteomic techniques. Their work, entitled "Immunocamouflage of latex surfaces by grafted methoxypoly(ethylene glycol) (mPEG): Proteomic analysis of plasma protein adsorption", was published in Science China Life Sciences, 2012, 55(3): 191-201.

The authors reported that covalent grafting of $\mathrm{mPEG}$ to latex beads gave rise to global immunocamouflage of the bead surface resulting in decreased plasma protein adsorption as assessed by flow cytometry, gel electrophoresis and advanced proteomic analysis using isobaric tags for relative and absolute quantitation (iTRAQ) labeling followed by mass spectrometry (MS) (Figure 1). The reduction in plasma adsorption occurred because of polymer-mediated thermodynamic steric exclusion and surface charge camouflage. The iTRAQ-MS findings also demonstrated that the grafted polymer prevented surface interactions with plasma proteins involved in the immune recognition of biomaterials and allogeneic cells.

Biologically, the reduced surface-plasma interactions would enhance biocompatibility by camouflaging antigenic sites and preventing the generation of immune recognition signals. The findings of this paper are supportive and predictive of previously reported observations of the immunocamouflage of RBCs and lymphocytes and of the prevention of viral invasion of cells. The knowledge derived from these proteomic studies will help to further define the physical and chemical parameters necessary for efficient immunocamouflage of allogeneic cells, tissues and biomaterials. The practical application of the immunocamouflage technology could have a significant impact on improving the safety of donor cells (e.g., RBCs), tissues and implantable biomaterials in clinical medicine.

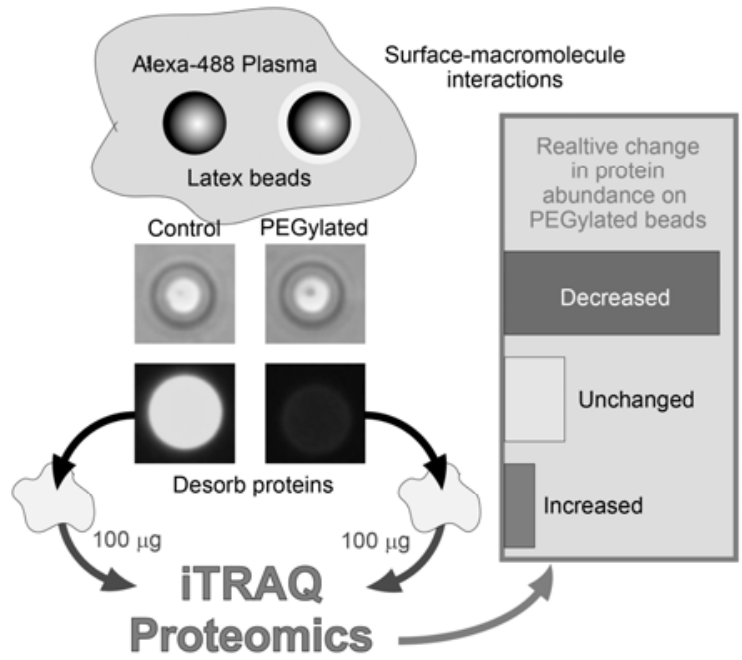

Figure 1 Schematic representation of the effects of immunocamouflage on protein adsorption. Qualitative and quantitative analysis of plasma surface interactions demonstrated the efficacy of immunocamouflage in preventing plasma protein adsorption. Moreover, iTRAQ analysis provided clear evidence for reduced adsorption of proteins associated with the immunological recognition of foreign cells/tissues and biomaterials.

See the article: Le Y, Li L, Wang D C, et al. Immunocamouflage of latex surfaces by grafted methoxypoly(ethylene glycol) (mPEG): Proteomic analysis of plasma protein adsorption. Sci China Life Sci, 2012, 55: 191-201

Open Access This article is distributed under the terms of the Creative Commons Attribution License which permits any use, distribution, and reproduction in any medium, provided the original author(s) and source are credited. 